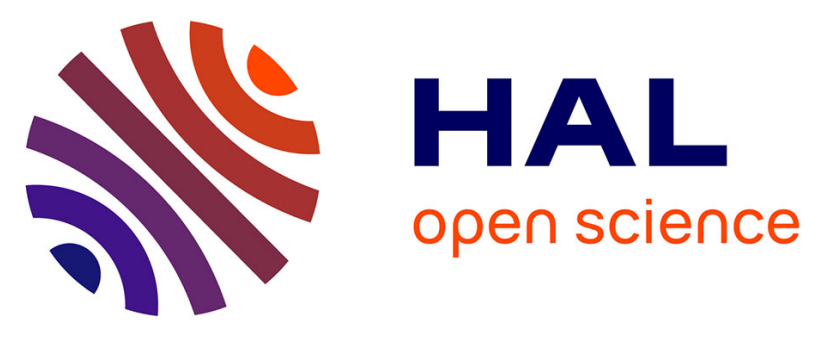

\title{
Relations entre anatomie, IRM et électrophysiologie dans la région sous-thalamique lors de l'implantation d'électrodes de stimulation cérébrale profonde pour maladie de Parkinson idiopathique sévère
}

Jérôme Coste, Lemlih Ouchchane, Philippe Derost, Miguel Ulla, Franck Durif, Jean Gabrillargues, Simone Hemm, Laurent Sarry, Jean-Jacques Lemaire

\section{To cite this version:}

Jérôme Coste, Lemlih Ouchchane, Philippe Derost, Miguel Ulla, Franck Durif, et al.. Relations entre anatomie, IRM et électrophysiologie dans la région sous-thalamique lors de l'implantation d'électrodes de stimulation cérébrale profonde pour maladie de Parkinson idiopathique sévère. Réunion de la Société de Neurochirurgie de Langue Française, Société de Neurochirurgie de Langue Française, Nov 2007, Paris, France. pp.418 - 419, 10.1016/j.neuchi.2007.09.017 . hal-01561357

\author{
HAL Id: hal-01561357 \\ https://hal.science/hal-01561357
}

Submitted on 31 Oct 2018

HAL is a multi-disciplinary open access archive for the deposit and dissemination of scientific research documents, whether they are published or not. The documents may come from teaching and research institutions in France or abroad, or from public or private research centers.
L'archive ouverte pluridisciplinaire HAL, est destinée au dépôt et à la diffusion de documents scientifiques de niveau recherche, publiés ou non, émanant des établissements d'enseignement et de recherche français ou étrangers, des laboratoires publics ou privés. 


\title{
Relations entre anatomie IRM et électrophysiologie dans la région sous-thalamique lors de l'implantation d'électrodes de stimulation cérébrale profonde pour maladie de Parkinson idiopathique sévère.
}

\author{
J Coste(1,2); L Ouchchane ${ }^{(3,4)}$; P Derost ${ }^{(6)} ;$ M Ulla(6), F Durif(6); J Gabrillargues ${ }^{(5)}$; \\ $S$ Hemm $^{(3)}$, L Sarry ${ }^{(3)}$ et JJ Lemaire ${ }^{(1,3)}$
}

${ }^{1}$ CHU Clermont-Ferrand, Hôpital Gabriel Montpied, Service de Neurochirurgie A, Clermont-Ferrand, F-63003 France.

2 INSERM, E216, Clermont-Ferrand, F-63000 France; Univ Clermont 1, UFR Odontologie, Laboratoire de Neurobiologie de la Douleur Trigéminale, Clermont-Ferrand, F-63000 France.

${ }_{3}^{3}$ INSERM, ERI14, Clermont-Ferrand, F-63001 France; Univ Clermont 1, UFR Médecine, Equipe de Recherche en Imagerie Médicale, Clermont-Ferrand, F-63000 France.

${ }^{4}$ CHU Clermont-Ferrand, Hôpital Gabriel Montpied, Laboratoire de Biostatistiques, Télématique et Traitement d'Images, Clermont-Ferrand, F-63003 France.

${ }^{5}$ CHU Clermont-Ferrand, Hôpital Gabriel Montpied, Service de Radiologie A, Clermont-Ferrand, F63003 France.

6 CHU Clermont-Ferrand, Hôpital Gabriel Montpied, Service de Neurologie, Clermont-Ferrand, F63003 France.

Introduction: Pour mieux comprendre les mécanismes de la stimulation cérébrale profonde (SCP) du noyau sous thalamique (NST) lors de la maladie de Parkinson idiopathique, nous avons étudié un des aspects, la relation entre anatomie IRM et activité neuronale spontanée.

Matériel et Méthodes: 38 trajectoires exploitables ont été analysées lors de 10 implantations bilatérales sous anesthésie locale. Les structures anatomiques ont été identifiées sur des images pondérées T2 (IRM 1,5 Tesla) et segmentées manuellement : principalement NST, Zona Incerta (Z1), Champs de Forel (H1, H2, pré-rubral) et Substance Noire (SN). L'activité neuronale spontanée a été systématiquement enregistrée sur $10 \mathrm{~mm}$ (pas : 0,5 mm), pendant 30 secondes, simultanément sur la trajectoire centrale (couvrant de façon optimale le NST) et une trajectoire antérieure $(+2 \mathrm{~mm}$ ). Nous avons contrôlé que les trajectoires ont suivi celles planifiées (radiographies per-opératoires, IRM post-opératoire). La SCP (trajectoire centrale: 17/20) a entrainé une amélioration de $63,6 \pm 6 \%$ du score UPDRS-III. Nous avons calculé la fréquence moyenne de décharge des neurones (FM) et la fréquence de survenue des burst (FB). 248 neurones (seuillage, analyse en composantes principales) ont été classés en fonction des structures anatomiques. Une analyse statistique a été effectuée (tests non paramétriques). 
Résultats : FM était différente entre ZI $(9,80 \pm 2,55 \mathrm{~Hz})$ et STN $(16,78 \pm 3,25), \mathrm{H} 2$ $(8,63 \pm 1,92)$ et STN, SN $(22,03 \pm 3,25)$ et STN et au sein du STN : dorso-latéral $(16,85 \pm 2,75)$ versus ventro-médian $(15.62 \pm 3.16)$.

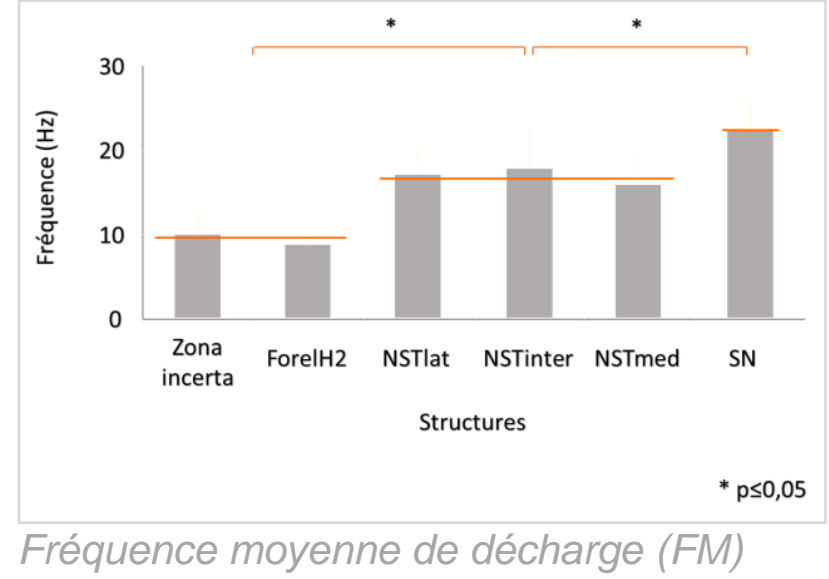

FB était plus élevée dans STN (32,67 $\pm 4,25$ burst.min-1) que dans les structures dorsales, Zl $(17,94 \pm 4,89)$ et $\mathrm{H} 2(9,63 \pm 2,54)$, ils différaient également de $\mathrm{SN}$ $(48,43 \pm 7,64)$.

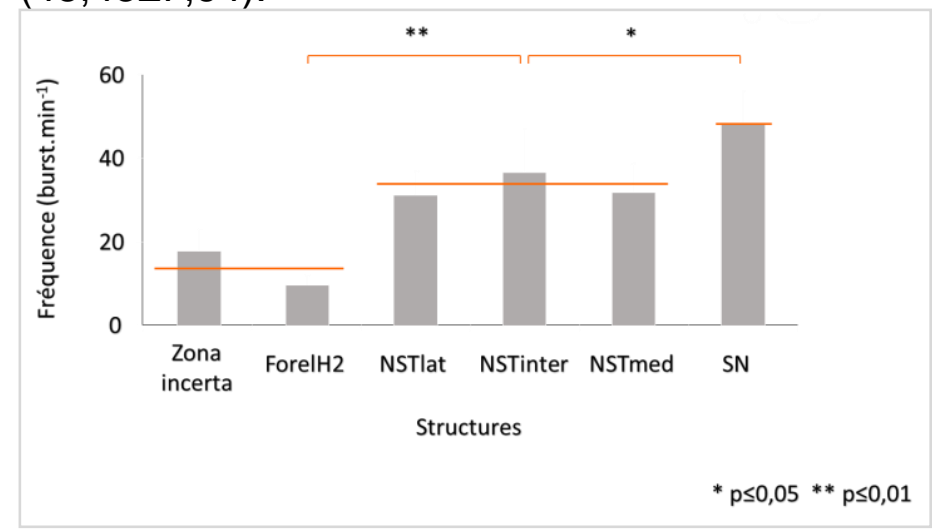

Fréquence de survenue des burst (FB)

Conclusions : II semble exister un lien entre les structures identifiées en IRM et l'activité cellulaire, cela même au sein du STN. L'analyse des résultats électrophysiologiques à partir de la littérature sera discutée. 